Journal of Mathematics and Statistics: 4(3): 174-180, 2008

ISSN: 1549-3644

(C) 2008 Science Publications

\title{
Block Methods based on Newton Interpolations for Solving Special Second Order Ordinary Differential Equations Directly
}

\author{
${ }^{1}$ Yap Lee Ken, ${ }^{2}$ Fudziah Ismail, ${ }^{2}$ Mohamed Suleiman and ${ }^{2}$ Suriah Md. Amin \\ ${ }^{1}$ Institute for Mathematical Research, University Putra Malaysia, \\ 43400, Serdang, Selangor, Malaysia \\ ${ }^{2}$ Departmen of Mathematics, University Putra Malaysia, 43400, Serdang, Selangor, Malaysia
}

\begin{abstract}
This study focused mainly on the derivation of the 2 and 3-point block methods with constant coefficients for solving special second order ordinary differential equations directly based on Newton-Gregory backward interpolation formula. The performance of the new methods was compared with the conventional 1-point method using a standard set of test problems. Numerical results were presented to illustrate the effectiveness of the methods in terms of total number of steps taken, maximum error and execution time. The results suggested a significant improvement in efficiency of the r-point block method. AMS Subject Classification: 65L05.
\end{abstract}

Key word: Initial value problems, special second order ordinary differential equations, block method

\section{INTRODUCTION}

Special second order Ordinary Differential Equations (ODEs) arises naturally in describing mechanics and electrical systems, wave oscillations and a variety of other physical problems. Such equations can be written in the form:

$$
\begin{array}{lr}
y^{\prime \prime}=f(x, y), & x \geq 0 \\
y\left(x_{0}\right)=y_{0}, & y^{\prime}\left(x_{0}\right)=y_{0}^{\prime}
\end{array}
$$

The easiest way to obtain the numerical solution of Eq. 1 is to reduce it to a system of first order ODEs twice the dimension. However some computational advantage can be gained if we can use methods specially designed to solve Eq. 1 directly, such methods can be seen in Dahlquist ${ }^{[1]}$, Van Der Houwen ${ }^{[9]}$ and Sommeijer $^{[8]}$ and El-Mikkawy and Rahmo ${ }^{[2]}$. These methods compute the numerical solution at one point at a time.

In this study we developed methods that can compute the numerical solution at more than one point at a time; such method is called block method. This method can be seen in Omar ${ }^{[6]}$ whereby he developed multiblock methods based on the divided difference interpolation for the solution of general second order equation $y^{\prime \prime}=f\left(x, y, y^{\prime}\right)$. Lee ${ }^{[4]}$ proposed block methods based on backward difference interpolation for first order ODEs $\mathrm{y}^{\prime}=\mathrm{f}(\mathrm{x}, \mathrm{y})$ and Majid ${ }^{[5]}$ developed the method based on Lagrange interpolation polynomial for general higher order ODEs $y^{d}=f\left(x, y, y^{\prime}, y^{\prime \prime}, \ldots, y^{d-1}\right)$.

In this study, we are going to derive the block method for solving special second order ODEs directly based on Newton-Gregory backward interpolation formula.

\section{MATERIALS AND METHODS}

Derivation of explicit r-point block method: In rpoint block method, the interval is divided into series of blocks with each block containing $r$ points; $r$ new values are obtained concurrently at each iteration of algorithm. Let $\mathrm{x}_{\mathrm{n}+\mathrm{i}}=\mathrm{x}_{\mathrm{n}}+\mathrm{ih}, \mathrm{i}=1,2, \ldots, \quad \forall \mathrm{n} \in[\mathrm{a}, \mathrm{b}]$. Therefore:

$\int_{x_{n}}^{x_{n+i}} \int_{x_{n}}^{x} y^{\prime \prime}(x) d x d x=\int_{x_{n}}^{x_{n+i}} \int_{x_{n}}^{x} f(x, y) d x d x$

Integrating Eq. 1 twice, we obtain the formula:

$y\left(x_{n}+i h\right)-y\left(x_{n}\right)=$ ihy $^{\prime}\left(x_{n}\right)+$
$\int_{x_{n}}^{x_{n+i}}\left(x_{n}+i h-x\right) f(x, y(x)) d x$

In order to eliminate the first derivative of $y(x)$, write the formula (2) with $\mathrm{h}$ replaced by $-\mathrm{h}$ and add the two expressions: 
$y\left(x_{n}+i h\right)-2 y\left(x_{n}\right)+y\left(x_{n}-i h\right)=$

$\int_{x_{n}}^{x_{n+i}}\left(x_{n}+i h-x\right)\left(f(x)+f\left(2 x_{n}-x\right)\right) d x$

which can be written as:

$$
\begin{aligned}
& y\left(x_{n+i}\right)-2 y\left(x_{n}\right)+y\left(x_{n-i}\right)= \\
& \int_{x_{n}}^{x_{n+i}}\left(x_{n+i}-x\right)\left(f(x)+f\left(2 x_{n}-x\right)\right) d x
\end{aligned}
$$

Define the interpolation polynomial $\mathrm{P}_{\mathrm{k}, \mathrm{n}}(\mathrm{x})$ which interpolates $\mathrm{f}(\mathrm{x}, \mathrm{y})$ at the $\mathrm{k}$ back values as follows:

$P_{k, n}\left(x_{n}+s h\right)=\sum_{q=0}^{k-1}(-1)^{q}\left(\begin{array}{c}-s \\ q\end{array}\right) \nabla^{q} f_{n}$

$P_{k, n}\left(x_{n}-s h\right)=\sum_{q=0}^{k-1}(-1)^{q}\left(\begin{array}{l}s \\ q\end{array}\right) \nabla^{q} f_{n}$

where, $s=\frac{x-x_{n}}{h}$.

Approximating $\mathrm{f}(\mathrm{x})$ and $\mathrm{f}\left(2 \mathrm{x}_{\mathrm{n}}-\mathrm{x}\right)$ with (4) and (5), (3) is now used in the form:

$$
\begin{aligned}
& y\left(x_{n+i}\right)=2 y\left(x_{n}\right)-y\left(x_{n-i}\right)+ \\
& \int_{x_{n}}^{x_{n+i}} \sum_{q=0}^{k-1}(-1)^{q}\left(x_{n+i}-x\right)\left[\left(\begin{array}{c}
-s \\
q
\end{array}\right)+\left(\begin{array}{l}
s \\
q
\end{array}\right)\right] \nabla^{q} f_{n} d x
\end{aligned}
$$

Replacing $\mathrm{dx}=\mathrm{hds}$ and changing the limit of integration in (6) gives:

$$
\begin{aligned}
& y\left(x_{n+i}\right)=2 y\left(x_{n}\right)-y\left(x_{n-i}\right)+ \\
& \int_{0}^{i} \sum_{q=0}^{k-1}(-1)^{q}(i-s) h\left[\left(\begin{array}{c}
-s \\
q
\end{array}\right)+\left(\begin{array}{l}
s \\
q
\end{array}\right)\right] \nabla^{q} f_{n} h d s
\end{aligned}
$$

which leads to:

$$
y_{n+i}=2 y_{n}-y_{n-i}+h^{2} \sum_{q=0}^{k-1} \omega_{i, q} \nabla^{q} f_{n}
$$

Where:

$$
\omega_{i, q}=(-1)^{\mathrm{q}} \int_{0}^{\mathrm{i}}(\mathrm{i}-\mathrm{s})\left[\left(\begin{array}{c}
-\mathrm{s} \\
\mathrm{q}
\end{array}\right)+\left(\begin{array}{l}
\mathrm{s} \\
\mathrm{q}
\end{array}\right)\right] \mathrm{ds}
$$

Let $\mathrm{V}_{\mathrm{i}}(\mathrm{t})$ be the generating function of the coefficients $\omega_{\mathrm{i}, \mathrm{q}}$ defined as follows:

$$
\begin{aligned}
\mathrm{V}_{\mathrm{i}}(\mathrm{t}) & =\sum_{\mathrm{q}=0}^{\infty} \omega_{\mathrm{i}, \mathrm{q}} \mathrm{t}^{\mathrm{q}} \\
& =\sum_{\mathrm{q}=0}^{\infty}(-\mathrm{t})^{\mathrm{q}} \int_{0}^{\mathrm{i}}(\mathrm{i}-\mathrm{s})\left[\left(\begin{array}{c}
-\mathrm{s} \\
\mathrm{q}
\end{array}\right)+\left(\begin{array}{c}
\mathrm{s} \\
\mathrm{q}
\end{array}\right)\right] \mathrm{ds} \\
& =\int_{0}^{\mathrm{i}}(\mathrm{i}-\mathrm{s}) \sum_{\mathrm{q}=0}^{\infty}\left((-\mathrm{t})^{\mathrm{q}}\left(\begin{array}{c}
-\mathrm{s} \\
\mathrm{q}
\end{array}\right)+(-\mathrm{t})^{\mathrm{q}}\left(\begin{array}{c}
\mathrm{s} \\
\mathrm{q}
\end{array}\right)\right) \mathrm{ds} \\
& =\int_{0}^{\mathrm{i}}(\mathrm{i}-\mathrm{s})\left((1-\mathrm{t})^{-\mathrm{s}}+(1-\mathrm{t})^{\mathrm{s}}\right) \mathrm{ds} \\
& =\frac{\left((1-\mathrm{t})^{\mathrm{i}}-1\right)^{2}}{(1-\mathrm{t})^{\mathrm{i}}[\ln (1-\mathrm{t})]^{2}}
\end{aligned}
$$

Hence:

$$
\left(\sum_{\mathrm{q}=0}^{\infty} \omega_{\mathrm{i}, \mathrm{q}} \mathrm{t}^{\mathrm{q}}\right)[\ln (1-\mathrm{t})]^{2}=-2+\frac{1}{(1-\mathrm{t})^{\mathrm{i}}}+(1-\mathrm{t})^{\mathrm{i}}
$$

Using the expansions, gives:

$$
\begin{gathered}
\left(\omega_{i, 0}+\omega_{i, 1} t+\omega_{i, 2} t^{2}+\ldots\right)\left(t^{2}+\frac{2}{3} h_{2} t^{3}+\frac{2}{4} h_{3} t^{4}+\ldots\right) \\
=\left[\frac{1}{2}(i)(i+1) t^{2}+\frac{1}{6}(i)(i+1)(i+2) t^{3}+\ldots\right]+ \\
{\left[\frac{1}{2}(i-1)(i) t^{2}-\frac{1}{6}(i-2)(i-1)(i) t^{3}+\ldots\right]}
\end{gathered}
$$

By comparing coefficients yields:

$$
\begin{aligned}
& \omega_{i, 0}=i^{2} \\
& \omega_{i, q}=\frac{(i+q+1)(i+q) \ldots(i+1)(i)}{(q+2) !}+ \\
& (-1)^{q} \frac{(i-q-1)(i-q) \ldots(i-1)(i)}{(q+2) !}-2 \sum_{r=0}^{q-1} \frac{\omega_{r} h_{q+1-r}}{q+2-r}
\end{aligned}
$$

with $\mathrm{q}=1,2, \ldots$.

By using the integration coefficients and NewtonGregory backward difference formula, we derive the explicit methods of order four as shown.

\section{Explicit 2-point block method:}

$$
\begin{aligned}
& {\left[\begin{array}{ll}
1 & 0 \\
0 & 1
\end{array}\right]\left[\begin{array}{l}
\mathrm{y}_{\mathrm{n}+1} \\
\mathrm{y}_{\mathrm{n}+2}
\end{array}\right] }=\left[\begin{array}{cc}
-1 & 2 \\
0 & 2
\end{array}\right]\left[\begin{array}{c}
\mathrm{y}_{\mathrm{n}-1} \\
\mathrm{y}_{\mathrm{n}}
\end{array}\right]+\left[\begin{array}{cc}
0 & 0 \\
0 & -1
\end{array}\right]\left[\begin{array}{c}
\mathrm{y}_{\mathrm{n}-3} \\
\mathrm{y}_{\mathrm{n}-2}
\end{array}\right]+ \\
& \mathrm{h}^{2}\left(\left[\begin{array}{cc}
-\frac{5}{12} & \frac{7}{6} \\
-\frac{20}{3} & \frac{20}{3}
\end{array}\right]\left[\begin{array}{c}
\mathrm{f}_{\mathrm{n}-1} \\
\mathrm{f}_{\mathrm{n}}
\end{array}\right]+\left[\begin{array}{cc}
-\frac{1}{12} & \frac{1}{3} \\
-\frac{4}{3} & \frac{16}{3}
\end{array}\right]\left[\begin{array}{l}
\mathrm{f}_{\mathrm{n}-3} \\
\mathrm{f}_{\mathrm{n}-2}
\end{array}\right]\right)
\end{aligned}
$$




\section{Explicit 3-Point block method:}

$$
\left.\begin{array}{rl}
{\left[\begin{array}{lll}
1 & 0 & 0 \\
0 & 1 & 0 \\
0 & 0 & 1
\end{array}\right]\left[\begin{array}{l}
\mathrm{y}_{\mathrm{n}+1} \\
\mathrm{y}_{\mathrm{n}+2} \\
\mathrm{y}_{\mathrm{n}+3}
\end{array}\right]=} & {\left[\begin{array}{ccc}
0 & -1 & 2 \\
-1 & 0 & 2 \\
0 & 0 & 2
\end{array}\right]\left[\begin{array}{c}
\mathrm{y}_{\mathrm{n}-2} \\
\mathrm{y}_{\mathrm{n}-1} \\
\mathrm{y}_{\mathrm{n}}
\end{array}\right]+\left[\begin{array}{ccc}
0 & 0 & 0 \\
0 & 0 & 0 \\
0 & 0 & -1
\end{array}\right]\left[\begin{array}{c}
\mathrm{y}_{\mathrm{n}-5} \\
\mathrm{y}_{\mathrm{n}-4} \\
\mathrm{y}_{\mathrm{n}-3}
\end{array}\right]} \\
+ & \left(\left[\begin{array}{ccc}
\frac{1}{3} & -\frac{5}{12} & \frac{7}{6} \\
\frac{16}{3} & -\frac{20}{3} & \frac{20}{3} \\
27 & -\frac{135}{4} & \frac{45}{2}
\end{array}\right]\left[\begin{array}{c}
\mathrm{f}_{\mathrm{n}-2} \\
\mathrm{f}_{\mathrm{n}-1} \\
\mathrm{f}_{\mathrm{n}}
\end{array}\right]\right. \\
+\left[\begin{array}{lll}
0 & 0 & -\frac{1}{12} \\
0 & 0 & -\frac{4}{3} \\
0 & 0 & -\frac{27}{4}
\end{array}\right]\left[\begin{array}{l}
\mathrm{f}_{\mathrm{n}-5} \\
\mathrm{f}_{\mathrm{n}-4} \\
\mathrm{f}_{\mathrm{n}-3}
\end{array}\right]
\end{array}\right]
$$

Derivation of implicit R-Point block method: Let $\mathrm{x}_{\mathrm{n}+\mathrm{i}}=\mathrm{x}_{\mathrm{n}}+\mathrm{ih}, \mathrm{i}=1,2, \ldots, \forall \mathrm{n} \in[\mathrm{a}, \mathrm{b}]$. Therefore:

$\int_{x_{n}}^{x_{n+i}} \int_{x_{n}}^{x} y^{\prime \prime}(x) d x d x=\int_{x_{n}}^{x_{n+i}} \int_{x_{n}}^{x} f(x, y) d x d x$

Using the same approach as in the previous section, we obtain:

$$
\begin{aligned}
& y\left(x_{n+i}\right)-2 y\left(x_{n}\right)+y\left(x_{n-i}\right)= \\
& \int_{x_{n}}^{x_{n+i}}\left(x_{n+i}-x\right)\left(f(x)+f\left(2 x_{n}-x\right)\right) d x
\end{aligned}
$$

The interpolating polynomials which interpolate $f(x, y)$ at the set of points $\left(x_{n+i-m}, f_{n+i-m}\right)$ for $\mathrm{m}=0,1,2, \ldots, \mathrm{k}$ as follows:

$P_{k, n+i}\left(x_{n}+(s+i) h\right)=\sum_{q=0}^{k}(-1)^{m}\left(\begin{array}{c}-s \\ q\end{array}\right) \nabla^{m} f_{n+i}$

and

$$
P_{k, n+i}\left(x_{n}+(-s-i) h\right)=\sum_{q=0}^{k}(-1)^{m}\left(\begin{array}{c}
s+2 i \\
q
\end{array}\right) \nabla^{m} f_{n+i}
$$

where $s=\frac{x-x_{n+i}}{h}$.

Approximating $f(x)$ and $f\left(2 x_{n}-x\right)$ with (8) and (9), (7) is now used in the form:

$$
\begin{aligned}
& y\left(x_{n+i}\right)=2 y\left(x_{n}\right)-y\left(x_{n-i}\right)+ \\
& \int_{x_{n}}^{x_{n+i}} \sum_{q=0}^{k}(-1)^{q}\left(x_{n+i}-x\right)\left[\left(\begin{array}{c}
-s \\
q
\end{array}\right)+\left(\begin{array}{c}
s+2 i \\
q
\end{array}\right)\right] \nabla^{q} f_{n+i} d x
\end{aligned}
$$

Replacing $\mathrm{dx}=\mathrm{hds}$ and changing the limit of integration in (10) gives:

$$
\begin{aligned}
& y\left(x_{n+i}\right)=2 y\left(x_{n}\right)-y\left(x_{n-i}\right)+ \\
& \int_{-i}^{0} \sum_{q=0}^{k}(-1)^{q}(-s) h\left[\left(\begin{array}{c}
-s \\
q
\end{array}\right)+\left(\begin{array}{c}
s+2 i \\
q
\end{array}\right)\right] \nabla^{q} f_{n+i} h d s
\end{aligned}
$$

which leads to:

$$
y_{n+i}=2 y_{n}-y_{n-i}+h^{2} \sum_{q=0}^{k} v_{i, q} \nabla^{q} f_{n+i}
$$

where

$$
v_{i, q}=(-1)^{q} \int_{-i}^{0}(-s)\left[\left(\begin{array}{c}
-s \\
q
\end{array}\right)+\left(\begin{array}{c}
s+2 i \\
q
\end{array}\right)\right] d s
$$

In order to obtain a useful recurrence relation for the coefficients $v_{i, q}$, the method of generating function is used. Let the generating function $L_{i}(t)$ be defined as follows:

$$
\begin{aligned}
\mathrm{L}_{\mathrm{i}}(\mathrm{t}) & =\sum_{\mathrm{q}=0}^{\infty} \mathrm{v}_{\mathrm{i}, \mathrm{q}} \mathrm{t}^{\mathrm{q}} \\
& =\sum_{\mathrm{q}=0}^{\infty}(-\mathrm{t})^{\mathrm{q}} \int_{-\mathrm{i}}^{0}(-\mathrm{s})\left[\left(\begin{array}{c}
-\mathrm{s} \\
\mathrm{q}
\end{array}\right)+\left(\begin{array}{c}
\mathrm{s}+2 \mathrm{i} \\
\mathrm{q}
\end{array}\right)\right] \mathrm{ds} \\
& =\int_{-\mathrm{i}}^{0}(-\mathrm{s}) \sum_{\mathrm{q}=0}^{\infty}\left((-\mathrm{t})^{\mathrm{q}}\left(\begin{array}{c}
-\mathrm{s} \\
\mathrm{q}
\end{array}\right)+(-\mathrm{t})^{\mathrm{q}}\left(\begin{array}{c}
\mathrm{s}+2 \mathrm{i} \\
\mathrm{q}
\end{array}\right)\right) \mathrm{ds} \\
& =\int_{-\mathrm{i}}^{0}(-\mathrm{s})\left((1-\mathrm{t})^{-\mathrm{s}}+(1-\mathrm{s})^{\mathrm{s}+2 \mathrm{i}}\right) \mathrm{ds} \\
& =\frac{\left((1-\mathrm{t})^{\mathrm{i}}-1\right)^{2}}{[\ln (1-\mathrm{t})]^{2}}
\end{aligned}
$$

Hence:

$$
\left(\sum_{q=0}^{\infty} v_{i, q} t^{q}\right)[\ln (1-t)]^{2}=1-2(1-t)^{i}+(1-t)^{2 i}
$$

Using the expansions, gives:

$$
\begin{aligned}
\left(v_{i, 0}\right. & \left.+v_{i, 1} t+v_{i, 2} t^{2}+\ldots\right)\left(t^{2}+\frac{2}{3} h_{2} t^{3}+\frac{2}{4} h_{3} t^{4}+\ldots\right) \\
= & {\left[-(i)(i-1) t^{2}+\frac{1}{3}(i)(i-1)(i-2) t^{3}+\ldots\right] } \\
& +\left[(i)(2 i-1) t^{2}-\frac{1}{3}(i)(2 i-1)(2 i-2) t^{3}+\ldots\right]
\end{aligned}
$$


By comparing coefficients yields:

$$
\begin{aligned}
& v_{i, 0}= i^{2} \\
& v_{i, q}=(-1)^{q+1} \frac{2(i-q-1)(i-q) \ldots(i-1)(i)}{(q+2) !}+ \\
&(-1)^{q} \frac{2(2 i-q-1)(2 i-q) \ldots(2 i-1)(i)}{(q+2) !} \\
& \quad-2 \sum_{r=0}^{q-1} \frac{v_{r} h_{q+1-r}}{q+2-r}
\end{aligned}
$$

with $\mathrm{q}=1,2, \ldots$.

With the integration coefficients and NewtonGregory backward difference formula, we derive the implicit block methods of order four.

\section{Implicit 2-Point block method:}

$$
\begin{aligned}
{\left[\begin{array}{ll}
1 & 0 \\
0 & 1
\end{array}\right]\left[\begin{array}{l}
\mathrm{y}_{\mathrm{n}+1} \\
\mathrm{y}_{\mathrm{n}+2}
\end{array}\right]=\left[\begin{array}{cc}
-1 & 2 \\
0 & 2
\end{array}\right]\left[\begin{array}{c}
\mathrm{y}_{\mathrm{n}-1} \\
\mathrm{y}_{\mathrm{n}}
\end{array}\right]+\left[\begin{array}{cc}
0 & 0 \\
0 & -1
\end{array}\right]\left[\begin{array}{l}
\mathrm{y}_{\mathrm{n}-3} \\
\mathrm{y}_{\mathrm{n}-2}
\end{array}\right]+\mathrm{h}^{2}\left(\left[\begin{array}{cc}
\frac{19}{240} & 0 \\
\frac{16}{15} & \frac{1}{15}
\end{array}\right]\left[\begin{array}{c}
\mathrm{f}_{\mathrm{n}+1} \\
\mathrm{f}_{\mathrm{n}+2}
\end{array}\right]\right.} \\
\left.+\left[\begin{array}{cc}
\frac{7}{120} & \frac{17}{20} \\
\frac{16}{15} & \frac{26}{15}
\end{array}\right]\left[\begin{array}{c}
\mathrm{f}_{\mathrm{n}-1} \\
\mathrm{f}_{\mathrm{n}}
\end{array}\right]+\left[\begin{array}{cc}
-\frac{1}{240} & \frac{1}{60} \\
0 & \frac{1}{15}
\end{array}\right]\left[\begin{array}{c}
\mathrm{f}_{\mathrm{n}-3} \\
\mathrm{f}_{\mathrm{n}-2}
\end{array}\right]\right)
\end{aligned}
$$

\section{Implicit 3-Point block method:}

$$
\begin{aligned}
{\left[\begin{array}{lll}
1 & 0 & 0 \\
0 & 1 & 0 \\
0 & 0 & 1
\end{array}\right]\left[\begin{array}{l}
y_{n+1} \\
y_{n+2} \\
y_{n+3}
\end{array}\right]=} & {\left[\begin{array}{ccc}
0 & -1 & 2 \\
-1 & 0 & 2 \\
0 & 0 & 2
\end{array}\right]\left[\begin{array}{c}
y_{n-2} \\
y_{n-1} \\
y_{n}
\end{array}\right]+\left[\begin{array}{ccc}
0 & 0 & 0 \\
0 & 0 & 0 \\
0 & 0 & -1
\end{array}\right]\left[\begin{array}{c}
y_{n-5} \\
y_{n-4} \\
y_{n-3}
\end{array}\right] } \\
& +h^{2}\left(\left[\begin{array}{ccc}
\frac{19}{240} & 0 & 0 \\
\frac{16}{15} & \frac{1}{15} & 0 \\
\frac{621}{40} & -\frac{117}{20} & \frac{117}{80}
\end{array}\right]\left[\begin{array}{l}
f_{n+1} \\
f_{n+2} \\
f_{n+3}
\end{array}\right]+\left[\begin{array}{ccc}
\frac{1}{60} & \frac{7}{120} & \frac{17}{20} \\
\frac{1}{15} & \frac{16}{15} & \frac{26}{15} \\
0 & \frac{657}{80} & -\frac{207}{20}
\end{array}\right]\left[\begin{array}{c}
f_{n-2} \\
f_{n-1} \\
f_{n}
\end{array}\right]\right. \\
& \left.+\left[\begin{array}{ccc}
0 & 0 & -\frac{1}{240} \\
0 & 0 & 0 \\
0 & 0 & 0
\end{array}\right]\left[\begin{array}{l}
f_{n-5} \\
f_{n-4} \\
f_{n-3}
\end{array}\right]\right)
\end{aligned}
$$

Test problems: To illustrate the effectiveness of the method, the existing 1-point method which was also based on Newton interpolation ${ }^{[3]}$, the 2-point and 3point block methods with order $\mathrm{k}=4$ are used to solve the following problems numerically.

Problem 1: $y^{\prime \prime}=-\omega^{2} y+\left(\omega^{2}-1\right) \sin x, \quad y(0)=1$, $\mathrm{y}^{\prime}(0)=1+\omega, \omega=10, \mathrm{x} \in[0,2]$.

Exact solution: $\mathrm{y}(\mathrm{x})=\cos (\omega \mathrm{x})+\sin (\omega \mathrm{x})+\sin \mathrm{x}^{[8]}$.
Problem 2: $\mathrm{y}_{1}^{\prime \prime}=-4 \mathrm{x}^{2} \mathrm{y}_{1}-\frac{2 \mathrm{y}_{2}}{\mathrm{r}}, \quad \mathrm{y}_{1}\left(\mathrm{x}_{0}\right)=0$, $y_{1}^{\prime}\left(x_{0}\right)=-\sqrt{2 \pi}, \quad y_{2}^{\prime \prime}=-4 x^{2} y_{2}+\frac{2 y_{1}}{r}, \quad y_{2}\left(x_{0}\right)=1$, $\mathrm{y}_{2}^{\prime}\left(\mathrm{x}_{0}\right)=0$, with $\mathrm{r}^{2}=\mathrm{y}_{1}^{2}+\mathrm{y}_{2}^{2}, \mathrm{x} \in\left[\sqrt{\frac{\pi}{2}}, 10\right]$.

Exact solution: $y_{1}(x)=\cos \left(x^{2}\right), y_{2}(x)=\sin \left(x^{2}\right)^{[2]}$.

Problem 3: $z^{\prime \prime}+z=0.001 e^{i x}, z(0)=1, z^{\prime}(0)=0.9995 i$, $\mathrm{z} \in \mathrm{C}, \mathrm{z}(\mathrm{x})=\mathrm{u}(\mathrm{x})+\mathrm{iv}(\mathrm{x}), \mathrm{u}, \mathrm{v} \in \mathfrak{R}, \mathrm{u}(\mathrm{x})=\cos (\mathrm{x})+0.0005 \mathrm{x}$ $\sin (\mathrm{x}), \mathrm{v}(\mathrm{x})=\sin (\mathrm{x})-0.0005 \mathrm{x} \cos (\mathrm{x})$.

We choose to solve the equivalent real problems $\mathrm{u}^{\prime \prime}+\mathrm{u}=0.001 \cos (\mathrm{x}), \quad \mathrm{u}(0)=1, \quad \mathrm{u}^{\prime}(0)=0$, $\mathrm{v}^{\prime \prime}+\mathrm{v}=0.001 \sin (\mathrm{x}), \quad \mathrm{v}(0)=0, \quad \mathrm{v}^{\prime}(0)=0.9995$, $\mathrm{x} \in[0,40 \pi]^{[7]}$.

It is the "almost periodic" problem with the theoretical solution represents motion on a perturbed circular orbit in the complex plane.

\section{RESULTS AND DISCUSSION}

Table 1-12 show the performance comparison of new methods and the existing method in terms of the total number of steps taken, maximum error and execution time. The performance of the new code is also compared to code by $\mathrm{Omar}^{[6]}$ as well. The new code is used to solve the special second order ODEs $y^{\prime \prime}=f(x, y)$ directly whereas the code by $\operatorname{Omar}^{[6]}$ is for the general second order ODEs $y^{\prime \prime}=f\left(x, y, y^{\prime}\right)$. The notations used in the Table 1-12 are as follows:
$\mathrm{h} \quad=$ Step size used
METHOD $=$ Method employed
TSTEP $=$ Total number of steps taken to obtain the solution
MAXERR = Magnitude of the maximum error of the computed solution
TIME = Execution time taken in microseconds (ms)
E1PN = Existing explicit 1-point method in ${ }^{[3]}$
E2PBN = The new explicit 2-point 1-block method
E3PBN $=$ The new explicit 3-point 1-block method
I1PN = Existing implicit 1-point method in ${ }^{[3]}$
I2PBN $=$ The new implicit 2-point 1-block method
I3PBN = The new implicit 3-point 1-block method
$\mathrm{E} 1 \mathrm{PO}=$ Explicit 1 -point method in Omar ${ }^{[6]}$
E2PBO = Explicit 2-point block method in Omar ${ }^{[6]}$
E3PBO $=$ Explicit 3-point block method in Omar ${ }^{[6]}$
$\mathrm{I} 1 \mathrm{PO}=$ Implicit 1 -point method in Omar ${ }^{[6]}$
$\mathrm{I} 2 \mathrm{PBO}=$ Implicit 2-point block method in $\mathrm{Omar}^{[6]}$
$\mathrm{I} 3 \mathrm{PBO}=$ Implicit 3-point block method in $\mathrm{Omar}^{[6]}$ 
J. Math. \& Stat., 4(3): 174-180, 2008

Table 1: Performance comparison between E1PN, E2PBN and E3PBN for solving Problem 1

\begin{tabular}{llrlr}
\hline $\mathrm{h}$ & METHOD & TSTEP & MAXERR & TIME \\
\hline $10^{-2}$ & E1PN & 200 & $1.04867(-2)$ & 690 \\
& E2PBN & 102 & $1.01941(-2)$ & 670 \\
\multirow{4}{*}{$10^{-3}$} & E3PBN & 69 & $9.17238(-3)$ & 656 \\
& E1PN & 2000 & $1.15576(-4)$ & 4422 \\
& E2PBN & 1002 & $9.12913(-5)$ & 4140 \\
$10^{-4}$ & E3PBN & 669 & $6.68441(-5)$ & 4015 \\
& E1PN & 20000 & $1.16663(-6)$ & 43710 \\
& E2PBN & 10002 & $9.17045(-7)$ & 41003 \\
$10^{-5}$ & E3PBN & 6669 & $6.67303(-7)$ & 40142 \\
& E1PN & 200000 & $1.19013(-8)$ & 435537 \\
& E2PBN & 100002 & $9.16555(-9)$ & 409839 \\
& E3PBN & 66669 & $6.70794(-9)$ & 400231 \\
\hline
\end{tabular}

Table 2: Performance comparison between E1PN, E2PBN and E3PBN for solving Problem 2

\begin{tabular}{llrlr}
\hline $\mathrm{h}$ & METHOD & TSTEP & MAXERR & TIME \\
\hline $10^{-2}$ & E1PN & 875 & $2.28442(-3)$ & 2790 \\
& E2PBN & 439 & $1.64625(1)$ & 2394 \\
& E3PBN & 294 & 2.57005 & 2426 \\
$10^{-3}$ & E1PN & 8747 & $6.11465(-6)$ & 25036 \\
& E2PBN & 4375 & $4.80547(-6)$ & 21490 \\
& E3PBN & 2918 & $4.40915(-6)$ & 20988 \\
$10^{-4}$ & E1PN & 87467 & $6.09090(-8)$ & 249919 \\
& E2PBN & 43735 & $4.78556(-8)$ & 212804 \\
& E3PBN & 29158 & $3.48057(-8)$ & 208928 \\
$10^{-5}$ & E1PN & 874669 & $2.53863(-9)$ & 2498769 \\
& E2PBN & 437336 & $9.65937(-10)$ & 2130344 \\
& E3PBN & 291559 & $8.22541(-10)$ & 2090620 \\
\hline
\end{tabular}

Table 3: Performance comparison between E1PN, E2PBN and E3PBN for solving Problem 3

\begin{tabular}{llrll}
\hline $\mathrm{h}$ & METHOD & TSTEP & MAXERR & TIME \\
\hline $10^{-2}$ & E1PN & 12567 & $1.16486(-4)$ & 40318 \\
& E2PBN & 6285 & $9.25841(-5)$ & 38059 \\
& E3PBN & 4191 & $6.80643(-5)$ & 37577 \\
$10^{-3}$ & E1PN & 125664 & $1.16492(-6)$ & 399714 \\
& E2PBN & 62834 & $9.15580(-7)$ & 377070 \\
& 3PBN & 890 & $6.66222(-7)$ & 372453 \\
$10^{-4}$ & E1PN & 1256638 & $1.18440(-8)$ & 4006061 \\
& E2PBN & 628321 & $9.36450(-9)$ & 3767598 \\
& 3PBN & 418882 & $6.70706(-9)$ & 3729101 \\
$10^{-5}$ & E1PN & 12566371 & $2.87949(-8)$ & 40027952 \\
& 2PBN & 6283187 & $1.69135(-8)$ & 37641623 \\
& 3PBN & 4188793 & $6.94793(-9)$ & 37251793 \\
\hline
\end{tabular}

Table 4: Performance comparison between I1PN, I2PBN and I3PBN for solving Problem 1

\begin{tabular}{llrlr}
\hline $\mathrm{h}$ & METHOD & TSTEP & MAXERR & TIME \\
\hline $10^{-2}$ & I1PN & 200 & $9.16935(-3)$ & 980 \\
& I2PBN & 102 & $7.24986(-3)$ & 945 \\
& I3PBN & 69 & $5.36151(-3)$ & 920 \\
$10^{-3}$ & I1PN & 2000 & $9.93031(-5)$ & 5942 \\
& I2PBN & 1002 & $7.48576(-5)$ & 5672 \\
& I3PBN & 669 & $5.02747(-5)$ & 5630 \\
$10^{-4}$ & I1PN & 20000 & $1.00021(-6)$ & 59156 \\
& I2PBN & 10002 & $7.50543(-7)$ & 56323 \\
& I3PBN & 6669 & $5.00710(-7)$ & 55158 \\
$10^{-5}$ & I1PN & 200000 & $1.01023(-8)$ & 591520 \\
& I2PBN & 100002 & $7.55203(-9)$ & 561423 \\
& I3PBN & 66669 & $5.01627(-9)$ & 549786 \\
\hline
\end{tabular}

Table 5: Performance comparison between I1PN, I2PBN and I3PBN for solving Problem 2

\begin{tabular}{llrlr}
\hline $\mathrm{h}$ & METHOD & TSTEP & MAXERR & \multicolumn{1}{c}{ TIME } \\
\hline $10^{-2}$ & I1PN & 875 & $2.52652(-3)$ & 3655 \\
& I2PBN & 439 & $2.48192(-3)$ & 3469 \\
& I3PBN & 294 & $7.12478(-3)$ & 3478 \\
$10^{-3}$ & I1PN & 8747 & $2.39947(-5)$ & 33067 \\
& I2PBN & 4375 & $3.10920(-5)$ & 31730 \\
& I3PBN & 2918 & $5.98919(-6)$ & 31220 \\
$10^{-4}$ & I1PN & 87467 & $3.51616(-7)$ & 329383 \\
& I2PBN & 43735 & $3.71797(-7)$ & 315150 \\
& I3PBN & 29158 & $2.61012(-8)$ & 310986 \\
$10^{-5}$ & I1PN & 874669 & $2.52969(-9)$ & 3296280 \\
& I2PBN & 437336 & $2.14713(-9)$ & 3152669 \\
& I3PBN & 291559 & $7.74633(-10)$ & 3100176 \\
\hline
\end{tabular}

Table 6: Performance comparison between I1PN, I2PBN and I3PBN for solving Problem 3

\begin{tabular}{llrlr}
\hline $\mathrm{h}$ & METHOD & TSTEP & MAXERR & TIME \\
\hline $10^{-2}$ & I1PN & 12567 & $1.16487(-4)$ & 54832 \\
& I2PBN & 6285 & $7.51354(-5)$ & 52640 \\
& I3PBN & 4191 & $5.06011(-5)$ & 51687 \\
$10^{-3}$ & I1PN & 125664 & $1.16492(-6)$ & 544661 \\
& I2PBN & 62834 & $7.49128(-7)$ & 520697 \\
& I3PBN & 41890 & $4.99765(-7)$ & 510612 \\
$10^{-4}$ & I1PN & 1256638 & $1.18440(-8)$ & 5439182 \\
& I2PBN & 628321 & $7.56804(-9)$ & 5198387 \\
& I3PBN & 418882 & $5.13160(-9)$ & 5099863 \\
$10^{-5}$ & I1PN & 12566371 & $2.87949(-8)$ & 54426979 \\
& I2PBN & 6283187 & $1.69537(-8)$ & 52040186 \\
& I3PBN & 4188793 & $7.02614(-9)$ & 51013287 \\
\hline
\end{tabular}

Table 7: Performance comparison between E2PBN, E3PBN and E2PBO, E3PBO for solving Problem 1

\begin{tabular}{llrl}
\hline $\mathrm{h}$ & METHOD & TSTEP & MAXERR \\
\hline $10^{-2}$ & E2PBN & 102 & $1.01941(-2)$ \\
& E2PBO & 102 & $4.69426(-2)$ \\
& E3PBN & 69 & $9.17238(-3)$ \\
$10^{-3}$ & E3PBO & 69 & $4.17711(-2)$ \\
& E2PBN & 1002 & $9.12913(-5)$ \\
& E2PBO & 1002 & $4.98878(-3)$ \\
& E3PBN & 669 & $6.68441(-5)$ \\
$10^{-4}$ & E3PBO & 669 & $4.98097(-3)$ \\
& E2PBN & 10002 & $9.17045(-7)$ \\
& E2PBO & 10002 & $4.99914(-4)$ \\
& E3PBN & 6669 & $6.67303(-7)$ \\
$10^{-5}$ & E3PBO & 6669 & $4.99906(-4)$ \\
& E2PBN & 100002 & $9.16555(-9)$ \\
& E2PBO & 100002 & $4.99992(-5)$ \\
& E3PBN & 66669 & $6.70794(-9)$ \\
& E3PBO & 66669 & $4.99992(-5)$ \\
\hline
\end{tabular}

Table 8: Performance comparison between E2PBN, E3PBN and E2PBO, E3PBO for solving Problem .2

\begin{tabular}{llrl}
\hline $\mathrm{h}$ & METHOD & TSTEP & MAXERR \\
\hline $10^{-2}$ & E2PBN & 439 & $1.64625(1)$ \\
& E2PBO & 439 & $3.31501(-2)$ \\
& E3PBN & 294 & 2.57005 \\
$10^{-3}$ & E3PBO & 294 & $8.66280(-2)$ \\
& E2PBN & 4375 & $4.80547(-6)$ \\
& E2PBO & 4375 & $1.09973(-3)$ \\
& E3PBN & 2918 & $4.40915(-6)$ \\
$10^{-4}$ & E3PBO & 2918 & $1.09963(-3)$ \\
& E2PBN & 43735 & $4.78556(-8)$ \\
& E2PBO & 43735 & $1.10003(-4)$ \\
& E3PBN & 29158 & $3.48057(-8)$ \\
$10^{-5}$ & E3PBO & 29158 & $1.10003(-4)$ \\
& E2PBN & 437336 & $9.65937(-10)$ \\
& E2PBO & 437336 & $1.10003(-5)$ \\
& E3PBN & 291559 & $8.22541(-10)$ \\
& E3PBO & 291559 & $1.10003(-5)$ \\
\hline
\end{tabular}


Table 9: Performance comparison between E2PBN, E3PBN and E2PBO, E3PBO for solving Problem 3

\begin{tabular}{llll}
\hline $\mathrm{h}$ & METHOD & TSTEP & MAXERR \\
\hline $10^{-2}$ & E2PBN & 6285 & $9.25841(-5)$ \\
& E2PBO & 6285 & $4.99211(-3)$ \\
& E3PBN & 4191 & $6.80643(-5)$ \\
$10^{-3}$ & E3PBO & 4191 & $4.98425(-3)$ \\
& E2PBN & 62834 & $9.15580(-7)$ \\
& E2PBO & 62834 & $4.99497(-4)$ \\
& E3PBN & 41890 & $6.66222(-7)$ \\
$10^{-4}$ & E3PBO & 41890 & $4.99489(-4)$ \\
& E2PBN & 628321 & $9.36450(-9)$ \\
& E2PBO & 628321 & $4.99501(-5)$ \\
& E3PBN & 418882 & $6.70706(-9)$ \\
$10^{-5}$ & E3PBO & 418882 & $4.99506(-5)$ \\
& E2PBN & 6283187 & $1.69135(-8)$ \\
& E2PBO & 6283187 & $4.99698(-6)$ \\
& E3PBN & 4188793 & $6.94793(-9)$ \\
& E3PBO & 4188793 & $5.00226(-6)$ \\
\hline
\end{tabular}

Table 10: Performance comparison between I2PBN, I3PBN and I2PBO, I3PBO for solving Problem 1

\begin{tabular}{llrl}
\hline $\mathrm{h}$ & METHOD & TSTEP & MAXERR \\
\hline $10^{-2}$ & I2PBN & 102 & $7.24986(-3)$ \\
& I2PBO & 102 & $1.42942(-2)$ \\
& I3PBN & 69 & $5.36151(-3)$ \\
$10^{-3}$ & I3PBO & 69 & $1.43029(-2)$ \\
& I2PBN & 1002 & $7.48576(-5)$ \\
& I2PBO & 1002 & $1.50554(-3)$ \\
& I3PBN & 669 & $5.02747(-5)$ \\
$10^{-4}$ & I3PBO & 669 & $1.50553(-3)$ \\
& I2PBN & 10002 & $7.50543(-7)$ \\
& I2PBO & 10002 & $1.51305(-4)$ \\
& I3PBN & 6669 & $5.00710(-7)$ \\
$10^{-5}$ & I3PBO & 6669 & $1.51305(-4)$ \\
& I2PBN & 100002 & $7.55203(-9)$ \\
& I2PBO & 100002 & $1.51381(-5)$ \\
& I3PBN & 66669 & $5.01627(-9)$ \\
& I3PBO & 66669 & $1.51381(-5)$ \\
\hline
\end{tabular}

Table 11: Performance comparison between I2PBN, I3PBN and I2PBO, I3PBO for solving Problem 2

\begin{tabular}{llll}
\hline $\mathrm{h}$ & METHOD & TSTEP & MAXERR \\
\hline $10^{-2}$ & I2PBN & 439 & $2.48192(-3)$ \\
& I2PBO & 439 & $3.28838(-3)$ \\
& I3PBN & 294 & $7.12478(-3)$ \\
$10^{-3}$ & I3PBO & 294 & $3.28838(-3)$ \\
& I2PBN & 4375 & $3.10920(-5)$ \\
& I2PBO & 4375 & $3.32651(-4)$ \\
& I3PBN & 2918 & $5.98919(-6)$ \\
$10^{-4}$ & I3PBO & 2918 & $3.32651(-4)$ \\
& I2PBN & 43735 & $3.71797(-7)$ \\
& I2PBO & 43735 & $3.33029(-5)$ \\
& I3PBN & 29158 & $2.61012(-8)$ \\
$10^{-5}$ & I3PBO & 29158 & $3.33029(-5)$ \\
& I2PBN & 437336 & $2.14713(-9)$ \\
& I2PBO & 437336 & $3.33049(-6)$ \\
& I3PBN & 291559 & $7.74633(-10)$ \\
& I3PBO & 291559 & $3.33048(-6)$ \\
\hline
\end{tabular}

The maximum error is defined as MAXERR $=\max _{1 \leq \mathrm{i} \leq \mathrm{TSTEP}}\left(\left|\mathrm{y}\left(\mathrm{x}_{\mathrm{i}}\right)-\mathrm{y}_{\mathrm{i}}\right|\right)$
Table 12: Performance comparison between I2PBN, I3PBN and I2PBO, I3PBO for solving Problem 3

\begin{tabular}{llll}
\hline $\mathrm{h}$ & METHOD & TSTEP & MAXERR \\
\hline $10^{-2}$ & I2PBN & 6285 & $7.51354(-5)$ \\
& I2PBO & 6285 & $1.51236(-3)$ \\
& I3PBN & 4191 & $5.06011(-5)$ \\
$10^{-3}$ & I3PBO & 4191 & $1.51236(-3)$ \\
& I2PBN & 62834 & $7.49128(-7)$ \\
& I2PBO & 62834 & $1.51238(-4)$ \\
& I3PBN & 41890 & $4.99765(-7)$ \\
$10^{-4}$ & I3PBO & 41890 & $1.51237(-4)$ \\
& I2PBN & 628321 & $7.56804(-9)$ \\
& I2PBO & 628321 & $1.51239(-5)$ \\
& I3PBN & 418882 & $5.13160(-9)$ \\
$10^{-5}$ & I3PBO & 418882 & $1.51243(-5)$ \\
& I2PBN & 6283187 & $1.69537(-8)$ \\
& I2PBO & 6283187 & $1.51435(-6)$ \\
& I3PBN & 4188793 & $7.02614(-9)$ \\
& I3PBO & 4188793 & $1.51964(-6)$ \\
\hline
\end{tabular}

\section{CONCLUSION}

The block and non-block methods based on Newton-Gregory backward interpolation formula are compared in terms of three parameters namely the total number of steps, the accuracy and the execution time. As the step size decreases, 2-point block and 3-point block methods reduce the total number of steps taken to almost one half and one third compared to 1-point method. These results are expected since the r-point block methods calculate the values of $y$ at $r$ point simultaneously compared to non-block methods.

The maximum error for explicit 3-point block method is slightly smaller compared to explicit 2-point block method which in turn smaller compared to explicit 1-point method for various values of $h$. In general, the implicit methods are more accurate than the explicit counterparts. The implicit block methods have better accuracy than the implicit non-block methods.

Both the explicit and implicit block methods seem to be superior to the non-block counterparts in term of the execution time taken to obtain the solution. The implicit methods require more time to generate the solution since it involved extra computations. As the step size becomes finer, the advantage of using block methods is more obvious.

Table 7-12 show the advantage of using the new codes over codes by Omar ${ }^{[6]}$ in term of accuracy. The increase in the accuracy is more obvious as the step size decreases. Thus, it can be concluded that the performance of both the explicit and implicit block methods based on Newton-Gregory backward interpolation formula is better in terms of the total number of steps taken, accuracy and execution time compared to the non-block methods and is more accurate compared to the existing block methods. 


\section{REFERENCES}

1. Dahlquist, 1978. On accuracy and unconditional stability of linear multistep methods for second order differential equations. BIT Numeric. Math., 18: 133-136. DOI: 10.1007/BF01931689

2. El-Mikkawy, M. and El-D. Rahmo, 2003. A new optimized non-FSAL embedded Runge-KuttaNystrom algorithm of orders 6 and 4 in 6 stages. Applied Math. Comput., 145: 33-43. DOI: 10.1016/S0096-3003(02)00436-8

3. Hairer, E., S.P. Norsett and G. Wanner, 1993. Solving Ordinary Differential Equations I (Nonstiff Problems). 2nd Edn., Springer-Verlag, New York, pp: 528. ISBN: 3540566708.

4. Lee, L.S., M. Suleiman, Z. Omar and F. Ismail, 2000. Two and three-point explicit block methods for first order ordinary differential equations. Proceedings of the International Conference on Mathematics and its Applications in the New Millenium, July 18-19, Renaissance Palm Garden Hotel Putra Jaya, Malaysia, pp: 481-490.

5. Majid, Z. and M. Suleiman, 2007. Implementation of four-point fully implicit block methods for solving ordinary differential equations. Applied Math. $\quad$ Comput., 184: 514-522. http://cat.inist.fr/?aModele $=$ afficheN\&cpsidt $=1851$ 9329.
6. Omar, Z., M. Suleiman, M.Y. Saman and D.J. Evans, 2002. Parallel r-point explicit block method for solving second order ODEs directly. Int. J. Comput. $\quad$ Math., 79: 289-298. http://cat.inist.fr/?aModele $=$ afficheN\&cpsidt $=1365$ 2392.

7. Papageorgiou, G., I.Th. Famwlis and Ch. Tsitouras, 1998. A P-stable singly diagonally implicit runge-kuttanystrom method. Numeric. Algorithm, 17: 345-353. DOI: $10.1023 / \mathrm{A}: 1016644726305$.

8. Sallam, S. and A.A. Karaballi, 1999. A continuous implicit nystrom method for solving ordinary second order initial value problems. Int. J. Comput. Math., 72: 189-198.

9. Van Der Houwen, P.J. and B.P. Sommeijer, 1987. Predictor-corrector for periodic second order initial value problems. IMA J. Numeric. Anal., 7: 407-422. http://cat.inist.fr/?aModele=afficheN\&cpsidt=7432630. 